\title{
Responsabilidad sanitaria: responsabilidad personal del médico vs. responsabilidad del sistema sanitario
}

\author{
Medical liability: professional liability vs. public health \\ service liability
}

\begin{abstract}
Resumen
La responsabilidad médica es inseparable de la asistencia sanitaria que hoy en día exige planteamientos desde el pragmatismo y la aceptación. Se hacen necesarios mecanismos incorporados al propio sistema sanitario que den una adecuada solución a situaciones difícilmente asimilables por el profesional y que representan un requerimiento social necesitado de respuesta eficaz.

La judicialización mediante la vía de la responsabilidad penal puede llegar a dar respuesta puntual en el mejor de los casos, pero no aporta soluciones prospectivas al problema. En los sistemas sanitarios actuales se tiende a la búsqueda de procedimientos extrajudiciales que, desde la despersonalización y la "desculpabilización", permitan un abordaje contextual que, además de indemnizar, plantee acciones de mejora del sistema aumentando la seguridad y confianza de pacientes y profesionales.
\end{abstract}

Palabras clave: Responsabilidad profesional médica. Mala praxis. Errores médicos.

\section{Abstract}

The medical liability is an inseparable phenomenon from the health care that today needs demands from the pragmatic approach and acceptance. Built-in mechanisms are necessary for the health system itself so it can give an adequate solution to difficult situations so that they can be assimilated by the professional and it also represents social requirements needed of an effective answer.

The prosecution by criminal liability, in the best of the situations, could be solved promptly, but don't provide prospective solutions at the problem. In the actual health care systems they tend to look for extrajudicial solutions, allowing a contextual approach that permits, in addition to compensation, actions to improve the system to increase safety and confidence patients and professionals

Key words: Medical Liability. Malpractice. Medical errors.

La exigencia de responsabilidad al ejercicio de la Medicina ha venido configurando actitudes motivadas por las repercusiones legales y porque una práctica inadecuada tiene consecuencias económicas ${ }^{1}$ y de gestión, a lo que hay que añadir un reflejo nada despreciable en las noticias de prensa que influye en la percepción de seguridad clínica por parte de los ciudadanos $^{2}$. Estas nuevas actitudes pueden entenderse como mejoras objetivas unas, respondiendo claramente al principio inmutable "primum non nocere", como es la adopción de estándares y protocolos; y otras, como la medicina defensiva, como reacción que genera en el profesional, que lo percibe como una situación de presión judicial para la que no dispone de recursos dentro de la organización sanitaria.

Esta "presión judicial" no es más que un reflejo del modelo de sociedad actual en el que la reivindicación de derechos y la idea de haber recibido un perjuicio resarcible suele ser exageradamente interpelada a la práctica sanitaria dentro del Sistema Público de

\section{Rodríguez Hornillo ${ }^{1}$ \\ C. De la Riva \\ Moreno $^{2}$}

A. Serratosa

Sánchez-lbargüen ${ }^{2}$

${ }^{1}$ Doctor en Medicina y Cirugía.

Serv. de Aseguramiento y Riesgos.

Servicio Andaluz de

Salud.

²Licenciado en

Medicina y Cirugía.

Serv. de Aseguramiento y Riesgos.

Servicio Andaluz de

Salud.

Correspondencia:

Dr. Manuel Rguez. Hornillo E-mail:

manuel.rodriguez.hornillo. sspa@juntadeandalucia.es

Fecha de recepción: 25. NOV.2010

Fecha de aceptación: 27.ABR.2011 
Salud. Esta puntual interpretación de derechos constituye el marco general que propicia el volumen de demandas/reclamaciones al que actualmente asistimos y que preocupa en todos los países de nuestro medio ${ }^{3}$.

Se ha estudiado la posibilidad de reducir el número de demandas/reclamaciones en relación con los efectos adversos sin poder establecer elementos predictivos racionales para la reducción de tales demandas/reclamaciones ${ }^{4}$. Desde hace tiempo se viene observando que el acontecimiento de efectos adversos no guarda relación con las reclamaciones por presunta mala práctica que terminan en indemnización ${ }^{5}$. En pocas de estas demandas/reclamaciones se aportan pruebas del error que se pretende ${ }^{6}$.

Este desfase entre la aparición de efectos adversos de los tratamientos y las demandas/reclamaciones motiva que sean situaciones difícilmente asimilables por el profesional sanitario asistencial, en el que generan intranquilidad e inseguridad, cediendo a estos requerimientos con medidas que no hacen sino complicar la praxis médica (aumento de la documentación, consentimientos complicados, etc.). En su lugar, se deberían articular estructuras dentro de la propia organización sanitaria que se ocupen de gestionar esta exigencia de responsabilidad, descargando la presión sobre el facultativo y el resto del personal asistencial, tales como servicios médico legales hospitalarios a los que tanto el personal asistencial como los usuarios en general pudiesen recurrir como un servicio asistencial más, asesorando preventivamente y, en su caso, resolviendo directamente las incidencias que ocasionan un perjuicio al paciente o que éste cree haber sufrido.

Aunque en nuestro medio existen diferentes vías de abordaje de la responsabilidad médica ${ }^{7}$, se pueden resumir en dos: la vía judicial, civil y penal, y la vía extrajudicial, en la que la responsabilidad patrimonial de la administración es su principal cauce al no estar aún muy implantados en nuestro medio los procedimientos de mediación, conciliación y arbitraje. Fundamentaremos el presente artículo en el análisis comparativo de la responsabilidad desde una perspectiva penal frente a la visión desde un punto de vista patrimonial, como vías más importantes de dilucidar la existencia de responsabilidad médica.

La primera, la exigencia de responsabilidad penal, da lugar a una "denuncia/querella" en vía judicial y la segunda origina una "reclamación" en vía administrativa. En definitiva, se trata de la responsabilidad personal derivada de la obligación que todos tenemos de responder de nuestros actos ante la ley, el cumplimiento de la lex artis en el caso del médico, frente a la responsabilidad de la administración sanitaria cuyos Servicios Públicos de Salud tienen la obligación de proporcionar una asistencia adecuada en tiempo y lugar al problema que presenta el paciente, algo que va más allá de la lex artis pues incluye cuestiones como la organización de la asistencia (citas, horarios, ubicaciones los centros y servicios...), cumplimiento de protocolos y guías de asistencia, disponibilidad de medios técnicos y personales, etc., sin olvidar el compromiso de la oferta asistencial establecida por normativa (plazos quirúrgicos, cartera de servicios, etc.).

De tal forma, la responsabilidad penal se configura como la responsabilidad personal de ser "buen profesional", mientras que a la administración sanitaria le corresponde ser un "buen servicio de salud", actualizado y competente. En esta última cuestión, los límites de exigencia se amplían conforme a las muchas competencias de un Servicio Público de Salud, en el que no todo son actos sanitarios personales. Sin embargo, frecuentemente se demanda al médico por unas actitudes o unos medios que no dependen de él ni están a su alcance, sino que son subsidiarios del Servicio de Salud, que es donde se debería dirigir el requerimiento indemnizatorio.

Moviéndonos en el campo de la responsabilidad de la Administración Sanitaria (responsabilidad patrimonial), el análisis de un potencial error es más extenso que cuando se intenta evaluar la responsabilidad personal del médico o responsabilidad penal. Son numerosos los casos archivados en vía penal, al considerarse una actuación adecuada a lex artis, que en vía administrativa son estimados, al entender que no se tomaron las decisiones adecuadas o que la asistencia no tuvo el nivel de excelencia exigible al sistema, por lo que el resultado dañoso no debe ser asumido por el paciente y procede resarcir.

\section{Caso 1}

Paciente de 32 años de edad, obeso, fumador e hipertenso que tras abuso de ingesta acude a servicio de urgencia de asistencia primaria con dolor precordial sin cortejo vegetativo. Tras realización de ECG que no mostró alteración, es remitido a su domicilio con la presunción diagnóstica de dolor de origen osteomuscular, prescribiéndose diclofenaco, nolotil y diazepan, y la indicación de volver si continúa el dolor. A las dos horas de la asistencia, el paciente fue encontrado cadáver en la cama. El estudio necrópsico, que incluyó la histopatología del corazón y la determinación de enzimas en plasma y líquido pericárdico, confirmó la existencia de infarto de miocardio. Se procedió a denuncia penal que fue sobreseída y posterior reclamación patrimonial que fue estimada, dando lugar a indemnización por esta vía. 


\section{Comentario del caso}

El dolor torácico es una de las causas más frecuentes de consulta en los servicios de urgencias, tanto hospitalarios como de atención primaria ${ }^{8}$. Pueden llegar a significar hasta el $11,9 \%$ de consultas médicas urgentes. De ellos, un $15 \%$ son debidos a un síndrome coronario agudo (SCA) demostrable por clínica y electrocardiograma (ECG), que se incrementan hasta un $35 \%$ adicional si se incluyen los síndromes coronarios agudos con normalidad inicial en el ECG. La dificultad de su diagnóstico estriba en que ${ }^{9}$ :

- Hay que diferenciar entre el dolor somático y el visceral, dado que éste es, frecuentemente, la expresión de procesos con riesgo vital.

- Muchas estructuras torácicas generan dolor con la misma localización, dado que la inervación sensitiva corresponde al mismo segmento espinal (dermatomas C2 a D6). Por ejemplo, el dolor de origen esofágico y el coronario.

- Se localizan en el mismo sitio y con clínica similar cuadros potencialmente graves y patologías banales.

- No siempre existe una buena correlación entre la intensidad del dolor y la gravedad de la patología causante.

- Dada la frecuencia de las patologías que lo originan, en algunos casos, pueden coexistir en el mismo paciente varias causas productoras de dolor torácico agudo.
- En un Servicio de Urgencia, además, la valoración de un dolor torácico agudo conlleva una serie de peculiaridades: suele llevar poco tiempo de evolución, careciendo de todas sus características semiológicas. Además, podemos encontrarnos con la ausencia de algunos síntomas o signos asociados que ayuden a su diagnóstico. Se dispone, en general, de poco tiempo para la valoración sistemática del paciente, siendo, con frecuencia, la primera vez que se le asiste, desconociendo sus antecedentes, no aportando casi nunca informes clínicos previos, debiéndose, no obstante, adoptar una decisión inmediata: ingreso, traslado a centro hospitalario, observación o alta. Y las pruebas complementarias disponibles en cada Servicio de Urgencia, cuando existen, son limitadas, por lo que la anamnesis y la exploración física adquieren su máximo valor y rentabilidad.

El dolor torácico representa un desafío para el profesional, que le obliga a realizar en un breve periodo de tiempo un diagnóstico al menos sindrómico del que se derivará una actitud terapéutica urgente. En el caso expuesto, la normalidad electrocardiográfica desvió la atención sobre la patología cardiaca subyacente, siendo una eventualidad ajena a mala praxis, imprudencia, impericia o negligencia, por lo que es razonable la no consideración de responsabilidad penal. Sin embargo, desde el punto de vista de la responsabilidad patrimonial, es deducible que sí se pudo hacer mejor y que en gran medida se trataba de una situación evitable. Una mayor atención a los antecedentes del paciente hubiese consolidado una

\begin{tabular}{|lll|}
\hline \multicolumn{3}{|c|}{$\begin{array}{c}\text { Responsabilidad penal } \\
\text { (y civil derivada) }\end{array}$} \\
\hline Acción u omisión & $\begin{array}{l}\text { Negligencia, imprudencia o } \\
\text { impericia personal }\end{array}$ & $\begin{array}{l}\text { Defecto asistencial. Acción u omisión imputable a la } \\
\text { Administración }\end{array}$ \\
\hline Daño & $\begin{array}{l}\text { Físico o psíquico: efectivo y y } \\
\text { evaluable }\end{array}$ & $\begin{array}{l}\text { Físico, psíquico, material, moral, pérdida de } \\
\text { oportunidad, etc. No soportable }\end{array}$ \\
\hline Nexo & De causalidad directa & $\begin{array}{l}\text { Causalidad directa o indirecta: responsabilidad } \\
\text { objetiva, nexo no comprobable, daño } \\
\text { desproporcionado, etc. }\end{array}$ \\
\hline $\begin{array}{l}\text { Desactivación o } \\
\text { antijuridicidad }\end{array}$ & Lex artis & \begin{tabular}{l} 
Inevitabilidad e imprevisibilidad \\
\hline $\begin{array}{l}\text { Parámetros de } \\
\text { valoración }\end{array}$
\end{tabular} \\
\hline $\begin{array}{l}\text { Consecuencias } \\
\text { posibles }\end{array}$ & Pegislación vigente & $\begin{array}{l}\text { Variables en función de la calidad asistencial, exigencia } \\
\text { al sistema, etc. }\end{array}$ \\
\hline
\end{tabular}

Tabla 1.

Comparativa entre responsabilidad penal y patrimonial. 
sospecha diagnóstica que justificase solicitar determinaciones enzimáticas 0 , al menos, la derivación del paciente a un servicio de urgencias hospitalario donde estas pruebas se pudiesen realizar, lo que en tal caso hubiese propiciado el diagnóstico de isquemia cardíaca.

En definitiva, los requisitos de responsabilidad penal se articulan tradicionalmente en tres pasos, a saber: una acción u omisión, generalmente mediada por negligencia, imprudencia o impericia; en segundo lugar, un daño efectivo y evaluable; y, en tercer lugar, la existencia de un nexo de causalidad entre ambos. Pero cuando se trata de valorar a la Administración sanitaria este esquema se complica debido a muchos otros aspectos intervinientes (Tabla 1).

En cambio, cuando se cuestiona una asistencia en responsabilidad patrimonial, el primer requisito, es decir la acción u omisión, frecuentemente es pluripersonal, lo que desdibuja la responsabilidad individual.

En la determinación de la responsabilidad de la administración, la concepción del daño es mucho más amplia. No nos referimos al perjuicio en sí (físico o psíquico), que es similar cualquiera que sea la circunstancia que lo provoque o los elementos causales que manejemos, sino que bajo la perspectiva patrimonial es mucho más amplia la idea de lo que no debe o no tiene obligación de soportar el paciente. Por ejemplo, si un paciente acude a un servicio de urgencia de primer nivel con alguna lesión, desde una herida necesitada de sutura a un dolor torácico susceptible de reanimación, si el profesional que le asiste posee un suficiente nivel de preparación y pone a disposición del paciente todos los medios a su alcance, éste tendrá obligación de soportar el posible resultado adverso que se obtenga desde un punto de vista penal, lo cual puede suponer desde una cicatriz poco estética en el caso de la sutura de la herida hasta el fallecimiento si las medidas de reanimación no son suficientes. En cambio, desde el punto de vista patrimonial, sí puede ser exigible a la administración que disponga de personal con determinado nivel de preparación o de medios más avanzados que permitan un mejor resultado que, en caso de ser adverso, no deba ser soportado por el paciente.

En cuanto al nexo, la amplitud de actos bajo supervisión de la Administración sanitaria permite la imputación de prácticamente todo lo que pase bajo su tutela, salvo que medie circunstancia que desactiva la antijuridicidad, lo cual de nuevo se muestra diferente en un entorno que en otro. Mientras que lo realizado bajo lex artis desestima cualquier pretensión penal, en patrimonial, a pesar de que los facultativos hayan actuado de esta conformidad, el desenvolvimiento de los hechos puede llegar a entenderse como asistencialmente defectuoso y sólo la inevitabilidad e imprevisibilidad del resultado impediría la responsabilidad de la Administración.

Especial contraste entre ambas concepciones suponen los parámetros que habilitan para comprender una situación como responsable, sea punible o resarcible. En el caso de la responsabilidad penal vienen marcados por la legislación vigente, la acción debe concebirse como delictiva en alguna de sus figuras, lo cual plantea un marco muy concreto y más estático. En cambio, en responsabilidad patrimonial el análisis vendrá señalado en función de la calidad asistencial que se pretende y la exigencia que se quiera hacer al sistema, no desde un punto de vista personal del que efectúa la valoración, sino desde la exigencia y calidad que el propio sistema ofrece 0 asegura poseer. Esto nos conduce a un campo de estudio más amplio, dinámico y evolutivo, con la necesidad de utilizar instrumentos comparativos para un análisis multifactorial de la asistencia: planes de salud, protocolos, guías de actuación, descripción de los procesos asistenciales integrados, recomendaciones asistenciales, normativas y circulares sobre seguridad, calidad, garantías, plazos o listas de espera, etc.

\section{Caso 2}

Paciente de 37 años de edad que, a las 17:43 horas, acudió a un Servicio de Urgencias hospitalario aquejado de cefalea, dolor de hombros que se irradia a cuello, mareos, hormigueos en manos y pies, pérdida de visión, pitidos en los oídos, así como mala audición. Fue diagnosticado de "cefalea tensional", siendo dado de alta por mejoría con la prescripción de analgesia, observación domiciliaria y control por médico de cabecera. A las 07:23 horas del día siguiente acude de nuevo con desorientación, alteración del comportamiento y sudoración profusa; no respondía a órdenes verbales ni dolorosas. Se diagnostica accidente cerebrovascular hemorrágico, siendo ingresado y falleciendo el 27/06/03.

\section{Comentario del caso}

Cuando un paciente con cefalea es atendido en urgencias, el principal objetivo es diferenciar si es de tipo orgánico, es decir, ocasionada por algún proceso concreto susceptible de profundizar en su diagnóstico, o estamos ante una cefalea primaria de carácter benigno. Para ello, se debe realizar una anamnesis detallada para determinar las características del dolor y un examen físico que debe consignar los 
signos vitales y un examen general y neurológico. La exploración neurológica se concretará sobre el nivel de conciencia, estado de las funciones mentales, signos meníngeos, fondo de ojo, pares craneales, asimetrías en la fuerza y sensibilidad, reflejos osteotendinosos, coordinación y marcha.

En cuanto al último aspecto diferenciador que planteamos, una vez dilucidada la responsabilidad, el resultado de la vía penal se traduce en condenas que simplemente resarcen lo actuado. En cambio, la vía patrimonial, además de indemnizar, posibilita en cualquiera de sus sentidos, estimatorio o no, emprender acciones de mejora que eviten la repetición del hecho.

Muchos otros son los aspectos que en penal son desestimados mientras que al análisis de la responsabilidad patrimonial suponen una asistencia no del todo correcta y pueden dar lugar a resarcimiento, tales como los problemas originados por la curva de aprendizaje, la aparición de complicaciones no estrictamente inherentes al procedimiento, las infecciones nosocomiales, las lesiones vasculares o nerviosas postquirúrgicas, etc., aunque estos eventos adversos puedan estar recogidos en el documento de consentimiento, lo cual no exime de responsabilidad, como erróneamente se interpreta en algunos sectores médicos asistenciales.

Uno de los aspectos que más fácilmente ilustra la diferencia en la consideración de responsabilidad es la "curva de aprendizaje", es decir, aquellas situaciones en las que la prestación de la asistencia se verifica por personas en fase de adiestramiento o durante la puesta a punto de una nueva técnica. En estas circunstancias es más frecuente la aparición de sucesos adversos evitables ${ }^{10}$ que originan demandas/reclamaciones. Aunque son actos "personales" que bajo cierto punto de vista podrían interpretarse como debidos a impericia, desde el punto de vista penal no suponen una incorrección, pero no así en patrimonial. La responsabilidad penal se ve diluida por cuanto la exigencia al profesional debe venir medida por su nivel de conocimiento o por el de un "profesional medio", siempre y cuando que se mueva dentro de sus competencias y la actuación se halle refrendada por el estado actual de la ciencia médica. Del análisis penal de un posible perjuicio cuando el profesional ha puesto todos los medios y conocimientos a su alcance para la curación del paciente, sólo puede deducirse el deber jurídico del paciente de soportar las consecuencias.

Sin embargo, como venimos sosteniendo, otro orden de cosas interviene cuando analizamos el caso desde un punto de vista patrimonial, pues la administración sanitaria sí tiene mayor obligación de aportar medios materiales y personales en óptimas condiciones para la consecución de los fines asistenciales que se procuran. Es decir, ante la administración sanitaria, el paciente no tiene obligación de soportar perjuicios derivados del perfeccionamiento de métodos o técnicas como frecuentemente sucede, por ejemplo, durante la optimización de técnicas de cirugía laparoscópica ${ }^{11}$ que actualmente vienen implementándose frente a la cirugía convencional. En resumen, desde el punto de vista penal se cumple la obligación de medios del profesional, pero no así de la administración sanitaria desde la óptica patrimonial.

Cada vez más se acepta que los posibles errores que puedan producirse durante un proceso asistencial, los mal llamados "errores médicos", se deben enfocar para su corrección y futura prevención desde un punto de vista impersonal. Una vez aceptado el viejo aforismo de que "to err is human", título de un trabajo ${ }^{12}$ que dio lugar a una nueva concepción del efecto adverso en Medicina y que condicionó nuevos planteamientos en lo que se ha venido en llamar la "seguridad del paciente", tema preferente de preocupación de la $\mathrm{OMS}^{13}$, la tendencia actual es hacia la objetivación de la responsabilidad, fijando la atención más en el daño, en cómo evitarlo y en cómo repararlo, que en la posible acción culpable de quién lo ocasiona. Se acepta que la mayoría de estos errores se deben a problemas multifactoriales, sean por defectos de organización de la compleja atención sanitaria de hoy en día, por la participación de varios profesionales en la resolución de un episodio concreto o por otras causas.

Para un mejor esclarecimiento de los hechos es importante desculpabilizar lo personal, entendiendo que cuando se produce un perjuicio a un paciente, aunque lo personal pueda tener su influencia, es en definitiva un fallo asistencial, un fallo del sistema que favorece ese "error personal". Incidir en la búsqueda de responsables personales y la punición personal en situaciones en las que cualquier profesional sanitario podría verse envuelto no contribuye al arreglo del problema, sino que muy al contrario determina la adopción de medidas defensivas que no responden a criterios de calidad científico-técnica ni a los de adecuación de recursos, tan necesarios ambos en la masificada asistencia sanitaria actual en la que no hay que olvidar que "sólo el que no ve pacientes no se equivoca".

Cierto es que la Medicina incide directamente sobre derechos de las personas que la hace susceptible de especial vigilancia judicial, con una amplia normativa legal que ya desde los años de estudiante es difícilmente digerida. Pero también es claro que una 
concepción impersonal del error nos lleva a una más fácil profundización del problema, no condicionando posturas que impiden aflorar las verdaderas causas que dan lugar a perjuicios evitables en los pacientes.

Es sólo bajo esta actual concepción desculpabilizadora en la que una investigación interna del propio sistema sanitario puede llegar a identificar las situaciones de riesgo para los pacientes y proponer medidas de mejora para evitarlas. En cambio, el requerimiento por vía penal siempre hará que los profesionales y el propio sistema retraiga información pues, aunque se respete la obligación de auxilio a la justicia, la reserva es la tendencia lógica y natural justificada por el propio derecho constitucional cuando sostiene que "todos tienen derecho... a no declarar contra sí mismo, a no confesarse culpables y a la presunción de inocencia"14.

Evidentemente, siempre existirá la posibilidad de actuación negligente, inexperta o descuidada necesitada de reprensión personal, pero no es lo frecuente. Después de gestionar en nuestro servicio unas 700 reclamaciones/año por vía administrativa, muchas de las cuales ya fueron archivadas en la vía penal, podemos afirmar sin temor a equivocarnos que el error imprudente con repercusión dañosa es infrecuente en la práctica asistencial, no así el perjuicio al paciente por defecto asistencial o error impersonal evitable, los cuales rondan el $20 \%$ de estas reclamaciones analizadas.

Sin embargo, los argumentos de los reclamantes inciden marcadamente sobre el aspecto penal. Cierto es que en la situación actual el "perjudicado" ve más conveniente la vía penal que la vía administrativa por razones que sería largo comentar. Sólo un profundo conocimiento de la vía administrativa patrimonial y una gran fluidez en la misma puede cambiar esta tendencia. Potenciar la resolución de los expedientes por vía administrativa en un tiempo prudencial y con suficientes garantías de imparcialidad, son las principales líneas de actuación que harían esta vía más atractiva a los reclamantes y sus representantes legales. Una mayor agilidad del procedimiento igualmente aportaría ventajas a los profesionales y al propio sistema, permitiendo identificar situaciones asistenciales susceptibles de mejora.

No somos los únicos en afirmar que el abordaje de los eventuales errores asistenciales no debe centrarse únicamente en las consecuencias financieras o judiciales, sino que debe llegar también a la cabecera del paciente, haciendo hincapié en la prevención, la comunicación abierta, una indemnización rápida en su caso y un aseguramiento por parte del Sistema Público de Salud de los costos de todo el proceso como medida de protección, tanto del paciente como de los profesionales implicados ${ }^{15}$.

Como hemos apuntado, la actuación inexperta, imprudente o negligente es claramente subsidiaria de reprensión penal. Pero en el complejo entorno asistencial, donde la información para la toma de decisiones casi nunca es completa y éstas se toman frecuentemente con un considerable nivel de incertidumbre, el error como "concepto equivocado", "acción desacertada" o "juicio falso" es una constante en el trabajo médico ${ }^{16}$. Por ello, una vez más, este error no debe analizarse desde el punto de vista personal con repercusión penal, lo que supondría asignarle una connotación ominosa que realmente no tiene en la mayoría de las ocasiones, sino que con un enfoque más pragmático, enfatizar en el "pensamiento en sistemas" más que en los errores individuales para buscar soluciones y garantizar la "seguridad del paciente". El objetivo es crear procesos para prevenir los errores humanos de los que la compleja práctica asistencial actual no está exenta.

El análisis de los casos desde el punto de vista de la responsabilidad patrimonial es más amplio y más rico en detalles, derivándose repercusiones para la mejora asistencial. Cuando este análisis se realiza desde la óptica penal, aunque no exento de dificultad, los elementos a valorar se concretan en saber si la actuación del profesional se engloba o no dentro de la lex artis, o si es equiparable a la de cualquier otro profesional medio en esas circunstancias, o si el resultado vino determinado por negligencia, dejadez o impericia en el actuar. El nivel de exigencia al sistema, sin embargo, es y debe ser más estricto, pues no se pretende un servicio sanitario "medio", sino de calidad, por lo que le es exigible un alto, y en ocasiones el máximo nivel de aplicación de los recursos asistenciales.

Este planteamiento permite considerar situaciones indemnizables todas aquellas que podríamos formular esquemáticamente como aquellas situaciones que "no deben producirse o no son razonables en un servicio asistencial de salud de calidad". Es decir, la vía patrimonial permite la creación de "estándares de responsabilidad". Situaciones que en otros órdenes pueden ser consideradas como complicaciones médicas sin lugar a responsabilidad, en vía patrimonial pueden ser situaciones responsables según los casos, v.gr. el error diagnóstico.

Al venir la responsabilidad patrimonial medida por la exigencia de calidad al Sistema Sanitario, podemos concretar qué situaciones podrán ser consideradas como indemnizables y cuáles no. Siendo el grado de calidad definible, podemos establecer patrimo- 
nialmente qué perjuicios podemos considerar como inherentes a la propia asistencia y cuáles no, dando lugar a situaciones indemnizables. Esto se entiende bien en la comparación de la asistencia pública con la privada, donde las diferentes condiciones permiten diferentes estándares de calidad, como por ejemplo los plazos de espera quirúrgicos.

Son ya abundantes los trabajos que defienden la necesidad de una reforma de los sistemas de gestión de reclamaciones, conscientes de que la actividad sanitaria es una fuente potencial de perjuicios prevenibles. Habiéndose mostrado la vía judicial insuficiente para tal fin, se defiende la instauración de otros enfoques más efectivos ${ }^{17,18,19}$. En algunos países, la crisis de la responsabilidad médica ha dado como resultado un aumento espectacular de las primas de seguros y un acceso reducido a los pacientes a cuidados especializados, en particular en ámbitos como la obstetricia y ginecología, neurocirugía y cirugía ortopédica ${ }^{20}$.

En estos trabajos es común la propuesta de un sistema de arbitraje. En nuestro país, el sistema de arbitraje tiene aún un grado de implantación discreto y sólo se encuentra presente en algunas provincias (Vizcaya, Madrid y Orense). Actualmente se está promoviendo la creación de la Agencia de Arbitraje Sanitario de Andalucía, por parte del Consejo Andaluz de Colegios de Médicos ${ }^{21}$.

Un modelo de gestión por vía administrativa que no se base en la culpa simplifica la determinación de la responsabilidad y elimina la dependencia del proceso judicial, pudiendo operar de forma más eficiente y predecible ${ }^{18}$. En nuestro medio, una rápida e imparcial gestión de la reclamación patrimonial por vía administrativa puede contribuir en gran medida a la satisfacción de los pacientes y a la tranquilidad de los profesionales.

Por lo tanto, de lo anteriormente expuesto se desprenden dos grandes conclusiones: en primer lugar, la necesidad de tender a la despersonalización de la responsabilidad médica, desligándola de la actuación personal para encuadrarla en un ámbito más amplio, que engloba no sólo al personal sanitario, sino también a toda la compleja organización sanitaria; y en segundo lugar, que el llamado "error médico" debe dejar de interpretarse desde un punto de vista punitivo y puramente indemnizatorio, para pasar a considerarse una herramienta preventiva creando nuevos y mejores patrones de calidad al analizar en mayor profundidad y con mayor rigor las actuaciones sanitarias para evitación de nuevos sucesos, todo ello sin olvidar el resarcimiento del daño causado. Es aquí donde proponemos la articulación de servicios médico legales dentro de las propias organizaciones sanitarias que descarguen la presión sobre los servicios asistenciales encargados de solucionar los conflictos y de estructurar las actuaciones que puedan prevenirlos en el futuro, instaurando nuevos patrones de responsabilidad sanitaria desligados de la responsabilidad personal y que aseguren una asistencia sanitaria de calidad progresiva.

\section{Bibliografía}

1. Carles M. Responsabilidad por una práctica médica inadecuada: una perspectiva económica. Gac Sanit. 2003; 17:494-503.

2. Mira JJ, Guilabert M, Ortíz L, Navarro IM, PérezJover MV, Aranaz JM. Noticias de prensa sobre errores clínicos y sensación de seguridad al acudir al hospital. Gac Sanit. 2010;24(1):33-9.

3. Capstick B. The future of clinical negligence litigation? BMJ 2004;328:457-60.

4. Morris JA Jr, Carrillo $Y$, Jenkins JM, Smith PW, Bledsoe S, Pichert J, et al. Surgical adverse events, risk management, and malpractice outcome: morbidity and mortality review is not enough. Ann Surg. 2003;237(6):844-51; discussion 851-2.

5. Brennan TA, Sox CM, Burstin HR. Relation between negligent adverse events and the outcomes of medicalmalpractice litigation. N Eng/ J Med. 1996; 335 (26): 1963-7.
6. Studdert DM, Mello MM, Gawande AA, Gandhi TK, Kachalia A, Yoon C, et al. Claims, errors, and compensation payments in medical malpractice litigation. N Engl J Med. 2006;354(19):2024-33.

7. Hernández Moreno J, Hernández Gil ML, Hernández Gil A. Responsabilidad por malpraxis médica: la vía extrajudicial. Cuad med forense. 2002;28:7-24.

8. Rivero Guerrero JA, Ruiz Ruiz MJ. Dolor Torácico. En: Manual de Urgencias y Emergencias. Disponible en: http://www.medynet.com/usuarios/jraguilar/Manual \% 20 de $\% 20$ urgencias $\% 20 y \%$ 20Emergencias/

9. Muñoz Ávila, JA, Torres Murillo JM, Calderón de la Barca Gázquez JM, García Criado El, Jiménez Murillo LM. Manejo del paciente con dolor torácico agudo en atención primaria. Semergen 1998;24(2):137-45.

10. Singh $H$, Thomas EJ, Petersen LA, Studdert DM Medical errors involving trainees: a study of closed malpractice claims from 5 insurers. Arch Intern Med. 2007;167(19):2030-6. 
11. McLean TR. Risk management observations from litigation involving laparoscopic cholecystectomy. Arch Surg. 2006;141(7):643-8; discussion 648.

12. Kohn LT, Corrigan JM, Donaldson MS (eds). To Err Is Human: Building a Safer Health System. Washington, DC: National Academy Press, 1999.

13. Organización Mundial de la Salud [Internet]. Disponible en: http://www.who.int/patientsafety/es/

14. Constitución Española. Artículo 24.2

15. Sage WM. Malpractice liability, patient safety, and the personification of medical injury: opportunities for academic medicine. Acad Med. 2006;81(9): 823-6.

16. Rodríguez L. La Clínica y su método. Reflexiones sobre dos épocas. Madrid: Díaz de Santos, 1999 $143 \mathrm{p}$.
17. Nigenda G, Ruiz JA, Monte J. Nuevas tendencias en la regulación de la profesión médica en el contexto de la reforma del sector salud: el caso de México. Rev Med Chil. 2001;129(11):1343-50.

18. Bovbjerg RR. Malpractice crisis and reform. Clin Perinatol. 2005;32(1):203-33, viii-ix.

19. Schoenbaum SC, Bovbjerg RR. Malpractice reform must include steps to prevent medical injury. Ann Intern Med. 2004;140(1):51-3.

20. Weinstein SL. Medical liability reform crisis 2008. Clin Orthop Relat Res. 2009;467(2):392-401. Epub 2008 Nov 7.

21. Diario Médico [Internet]. Disponible en: http://www.diariomedico.com/2010/09/01/areaprofesional/normativa/agencia-andaluza-paraarbitrar-danos. 